\title{
Eine Bereicherung der Psychiatriegeschichte
}

Iris Ritzmann

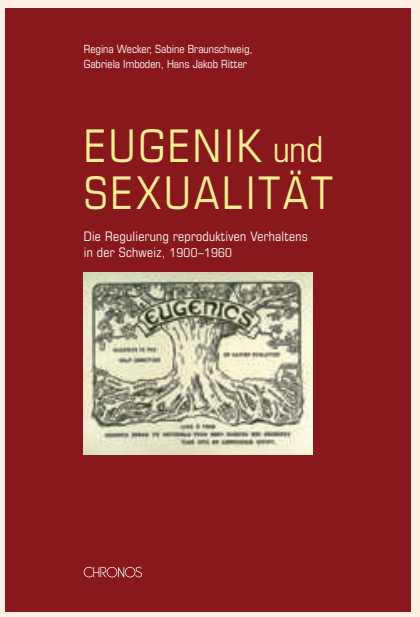

Regina Wecker, Sabine Braunschweig, Gabriela Imboden, Hans Jakob Ritter Eugenik und Sexualität

Die Regulierung reproduktiven Verhaltens in der Schweiz 1900-1960.

Zürich: Chronos Verlag; 2013.

201 Seiten. 38 CHF

ISBN 978-3-0340-1131-0
Ein differenziertes Bild skizziert Gabriela Imboden in ihrer Untersuchung der Kastration als einem Eingriff zur Regulierung männlicher Sexualität. Sie weist nach, dass sich zwar die Toleranz gegenüber ausserehelichem Geschlechtsverkehr im Verlauf des 20. Jahrhunderts vergrösserte, diese Entwicklung aber nicht zu einer generellen Liberalisierung sämtlicher sexueller Praktiken geführt habe. Zudem stellt sie einen geschlechtsspezifischen Unterschied in den sexualpsychiatrischen Eingriffen fest: Während bei Frauen mit der Sterilisierung primär eine «unerwünschte Nachkommenschaft» verhindert werden sollte, richtete sich die Kastration von Sexualstraftätern gegen sexuelle Handlungen per se.

Auf eine analoge Rollenverteilung kommt auch die Untersuchung von Imboden und Hans Jakob Ritter zur Abtreibung und Sterilisierung, die bei Männern eine «gefährliche Sexualität», bei Frauen eine «sexuelle Gefährdung» ausmacht. Besonders facettenreich sind die Ergebnisse einer weiteren Studie von Imboden und Ritter zur Ehefähigkeitsbegutach-

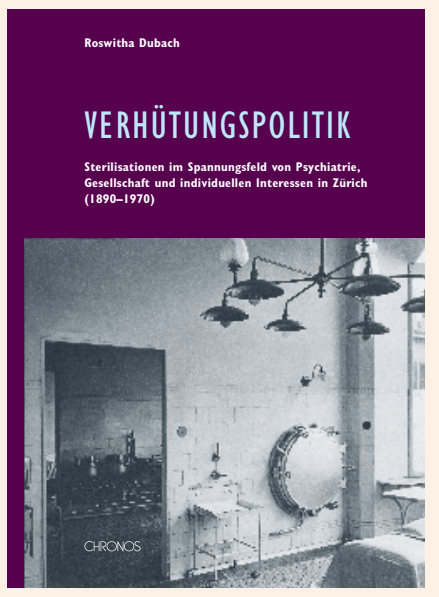

\section{Roswitha Dubach}

Verhütungspolitik

Sterilisationen im Spannungsfeld von Psychiatrie, Gesellschaft und individuellen Interessen in Zürich (1890-1970).

Zürich: Chronos Verlag; 2013.

351 Seiten. $48 \mathrm{CHF}$

ISBN 978-3-034-01134-1 schen Argumente der Eugenik die Wahrnehmung von Sexualität? Könnte die Eugenik sogar eine gebefördert haben? Inwiefern beeinflusste die Eugenik die Sexual- und Geschlechterpolitik? Diesen Fragen widmet sich der Sammelband «Eugenik und Sexualitat» von Regina Wecker, Sabine Braunschweig, Gaanderen Publikationen im Umfeld des Nationalfondsprojekts «Integration und Ausschluss» mitgewirkt haben. Basierend auf Basler Krankenakten Themen der institutionellen Sexualitätsregulierung Friedmatt herausarbeiten, dass sexuelle Bedürfniss von Patientinnen und Patienten einen ständigen ten bildeten, dem mit medikar ven Eingriffen begegnet wurde. 
tung. Mit der Umsetzung des Eheverbots für Geisteskranke 1912 löste die medizinisch-psychiatrische Begutachtung die zuvor ökonomisch-moralische Überprüfung von Ehewilligen ab. Um der sittlichen und eugenischen Gefahr entgegenzutreten, dass Paare nach einem Eheverbot im Konkubinat lebten und uneheliche Kinder zeugten, etablierte sich die Praxis der «sterilen Ehe», der Verknüpfung eines Ehefähigkeitsgutachtens mit einer Sterilisationsforderung, was die Familiengründung als traditionelles Ziel einer ehelichen Beziehung erschütterte. Das Selbstverständnis der Eugenik als moderner Praxis, die zu einer Liberalisierung der Sexualität führe, lässt vor dem jeweiligen politisch-rechtlichen Hintergrund mit unterschiedlichen Motiven verbunden wurden. In den ersten Jahrzehnten des Untersuchungszeitraums wurden nur vereinzelt Sterilisationen durchgeführt, meist jedoch mit erzwungener Einwilligung. In den 1920er und 30er Jahren war die Zürcher Poliklinik eine Anlaufstelle für ungewollt schwangere Frauen, die sich in einer sozialen Notlage befanden und abtreiben wollten. Diese Frauen willigten nur deshalb in die Sterilisation ein, weil der Eingriff zur Bedingung einer Abtreibung gemacht wurde. Bei einem Drittel der Sterilisationsentscheide kamen eugenische Argumente zur Sprache.

\section{Eugenik wird gedeutet als Machtinstrument gegen ärmere Bevölkerungsschichten und als Erweiterung einer biopolitischen Wirkkraft von Wissenschaft und Medizin.}

sich zumindest an diesem Beispiel der «sterilen Ehe» in manchen Punkten nachvollziehen.

In einem fünften Beitrag stellt Regina Wecker den Diskurs über Sexualität, Verhütung und Sterilisation in einen gesellschaftlichen Gender-Kontext. Vertieft geht sie dabei insbesondere auf die konkrete Nachfrage nach Verhütungswissen, die damaligen Praktiken, aber auch die Langzeitfolgen von weiblicher Sterilisation ein. Eugenik wird von den Herausgeberinnen vorerst als revolutionäres Moment
Für die Nachkriegszeit weist Dubach eine Individualisierung der Sterilisationspraxis nach, die nun auch vermehrt Männer einbezog. Eine veränderte Gesetzeslage und die Verfügbarkeit der Antibabypille beschleunigten den Übergang zu einer selbstbestimmten Familienplanung. Der Anteil eugenisch begründeter Sterilisationen schrumpfte bis in die 1960er Jahre auf ein Fünftel zusammen. Diesen Befund hinterfragt Dubach allerdings mit der These einer «Verschleierung eugenischer Motive», da weiterhin

\section{Beide Bücher realisieren eine patientennahe Psychiatriegeschichte auf der Basis von psychiatrischen Krankenakten.}

im Kontrast zur gängigen Interpretation aufgeworfen und diskutiert, im Fazit dann allerdings deutlich als Machtinstrument gegen ärmere Bevölkerungsschichten und zur Erweiterung einer biopolitischen Wirkkraft von Wissenschaft und Medizin gedeutet.

Auch die Historikerin Roswitha Dubach widmet sich in ihrer Monographie «Verhütungspolitik» der Verknüpfung von Sexualität, Biopolitik und Psychiatrie. Die sorgfältig recherchierte Arbeit, der eine umfangreiche Dissertation zugrunde liegt, untersucht die Interessen hinter der Sterilisationspraxis zwischen 1890 und 1970 am Beispiel der Zürcher Psychiatrischen Poliklinik und der Psychiatrischen Universitätsklinik «Burghölzli».

Dubach kann unterschiedliche Zeitabschnitte voneinander abgrenzen, in denen die Sterilisationen
Sterilisationen geistig behinderter Personen durchgeführt wurden, nun aber therapeutische Überlegungen oder das Kindswohl als Begründungen auftauchten.

Beide Werke bereichern die neuere Schweizer Psychiatriegeschichte um ein wichtiges Kapitel, den bevölkerungspolitischen und institutionellen Umgang mit Sexualität und Fortpflanzung. Auch methodisch weisen die Publikationen Analogien auf: Sie realisieren eine patientennahe Psychiatriegeschichte auf der Basis von psychiatrischen Krankenakten, deren Potential als historische Quelle auch mit diesen gehaltvollen Analysen noch lange nicht ausgeschöpft ist. Und schliesslich regen beide Bücher dazu an, heutige Normvorstellungen von Sexualität als Resultat historischer Prozesse zu begreifen und auf ihre Auswirkungen hin kritisch zu hinterfragen. 